\title{
A LIST OF ERRORS IN TABLES OF THE PELL EQUATION
}

BY D. H. LEHMER

Five tables of solutions of the Pell equations

$$
\begin{aligned}
& x^{2}-A y^{2}=1, \\
& x^{2}-A y^{2}=-1,
\end{aligned}
$$

have been published giving solutions* from $A=2$ to $A=1700$. Arranged in chronological order these tables are as follows:

1. A. M. Legendre, Thêorie des Nombres, Paris, 1798, Table xii.

2. C. F. Degen, Canon Pellianus etc., Copenhagen, 1817.

3. A. M. Legendre, Théorie des Nombres, 3d ed., 1830, Table $\mathrm{x}$.

4. C. E. Bickmore, British Association Report, vol. 53 (1893), p. 73.

5. E. E. Whitford, The Pell Equation, New York, 1912, p. 102.

The scope of each of the tables is as follows:

1, 3 Legendre, $A=2$ to 1003 . Gives solutions of (2) when possible, otherwise of (1).

2. Degen, $A=2$ to 1000 . Gives solutions of (1) and also of (2) when possible.

4. Bickmore, $A=1001$ to 1500 . Gives solutions of (2) when possible, otherwise of (1).

5. Whitford, $A=1501$ to 1700 . Gives solutions of (1) and also of (2) when possible.

A detailed account of these tables together with a list of errors in Legendre's and Degen's tables is included in an article by A. J. C. Cunningham. $\dagger$ This list, obtained

* In the present paper, by "solution" we mean "fundamental solution." $\dagger$ Messenger of Mathematics, vol. 46, p. 49. 
evidently by reading the tables together, is incomplete and not altogether correct.

The five tables mentioned above have been examined and thoroughly checked by the present writer. It is believed that no error has escaped detection.

The ordinary method of verifying a solution consists in actually substituting the quantities $x$ and $y$ in the equation. Cayley remarks that it is easier to calculate $A y^{2} \pm 1$ and then show that the square root of this quantity is actually $x$. Although this method is very laborious when $x$ and $y$ are large, one would naturally suppose that in using this check all errors would be detected. This however seems not to be the case. Non-typographical errors appear in the tables of Bickmore and Whitford though both tables were submitted to this check before publication. It is significant that these errors occur in solutions which are unusually large.

In obtaining the following list of errors a different method of checking was used in the case of solutions consisting of more than five or six digits. Instead of substituting the actual values of $x$ and $y$ in the equation, their remainders on division by some modulus were used. Three different moduli were employed, namely, 1001, 10001, and 100001, depending on the printing in the table to be checked. These moduli, chosen for their ease in casting out, made the checking extremely rapid and practically independent of the size of the solution. The necessary calculations were made on an ordinary computing machine without putting pen to paper.

Although this method does not afford as complete a check as would be obtained by using the actual values of $x$ and $y$, the chance of a compensating error is certainly negligible when using these large moduli.

In order to save space and avoid confusion only the corrected entries corresponding to arguments in which errors have been made are given below. Also uniform notation is used, the equation being taken as

$$
x^{2}-A y^{2}= \pm 1 \text {. }
$$


There are several points of interest to be noted. In the first edition of Legendre's table for every argument $A<250$ which has an error in $x$, the same error is made in the $x$ corresponding to the argument $4 A$. This is explained as follows. If in solving the equation for the argument $A$, the value for $y$ is found to be even $(2 n)$ we can write at once

$$
x^{2}-A(2 n)^{2}=x^{2}-4 A n^{2}= \pm 1 .
$$

The solution for $4 A$ was therefore obtained by merely dividing the $y$ by 2 and using the original $x$. This division by 2 was overlooked in the case of $A=344$, the entry given being identical with that for $A=86$. In general it can be shown that the solution for the argument $p^{2} A$ can be obtained from a fundamental or multiple solution for the argument $A$. This theorem seems not to have been noticed or at least not used to advantage by the workers on the Pell equation. For numbers containing a square factor greater than unity (there are 627 such $<1700$ ) the expansion of $\sqrt{A}$ in a continued fraction and the calculation of the successive convergents could have been avoided entirely.

Before publishing the $3 \mathrm{~d}$ edition of Legendre's table, the table in the first edition was compared with that of Degen. Nevertheless one error was carried over from the first edition and two from Degen's table. The other errors are for the most part typographical. There are two unimportant errors not listed below in Bickmore's table. For $A=1051$ the values of $x$ and $y$ are interchanged and the argument $A=1361$ should be printed with an asterisk $\left(1361^{*}\right)$ to designate equation (2). There are several errors of little consequence in the introductory table. Whitford's table contains one insignificant error; the argument 1589 is printed 1599. In Appendix A of Whitford's book are given the partial quotients and denominators of the complete quotients occurring in the expansion of $\sqrt{A}$ in a continued fraction from $A=1501$ to $A=2012$. In preparing a table 
of solutions of the Pell equation for $A=1701$ to $A=2000$, the present writer has found the following errors in the former table.

A

1733* The 6th partial quotient should be 3 and not 2 .

1822 The 23d partial quotient and denominator of the 23d complete quotient are missing. They are 1 and 54 respectively.

1852 The 29th partial quotient should be 20 and not 16 . 1963 The entry here should be:

$\begin{array}{rrrrrrrrrrr}44 & 3 & 3 & 1 & 2 & 3 & 2 & 29 & 9 & 1 & 4 \\ & 27 & 22 & 51 & 29 & 23 & 38 & 3 & 9 & 66 & 17\end{array}$

The above list comprises all the $A$ 's between 1700 and 2000 for which there is an error in a partial quotient.

It should be stated that in the tables of Degen, Bickmore, and Whitford which give the above mentioned elements of the expansion of $\sqrt{A}, A<1701$, only the solutions of the Pell equation were checked.

A

1. Legendre, 1 st edition, Table xii.

$$
\begin{aligned}
& 133 x=2588599 \\
& 214 x=695359189925 \\
& y=47533775646 \\
& 236 x=561799 \\
& 301 y=339113108232 \\
& 307 x=88529282 \\
& 331 x=2785589801443970 \\
& 343 x=130576328 \\
& y=7050459 \\
& 344 y=561 \\
& 355 y=50676 \\
& 365 x=3458 \\
& 397 x=20478302982 \\
& y=1027776565 \\
& 526 x=84056091546952933775 \\
& y=3665019757324295532 \\
& 532 x=2588599 \\
& 613 x=481673579088618
\end{aligned}
$$

* This error was also found by Cunningham, loc. cit. 
A

1. Legendre, 1st edition-continued

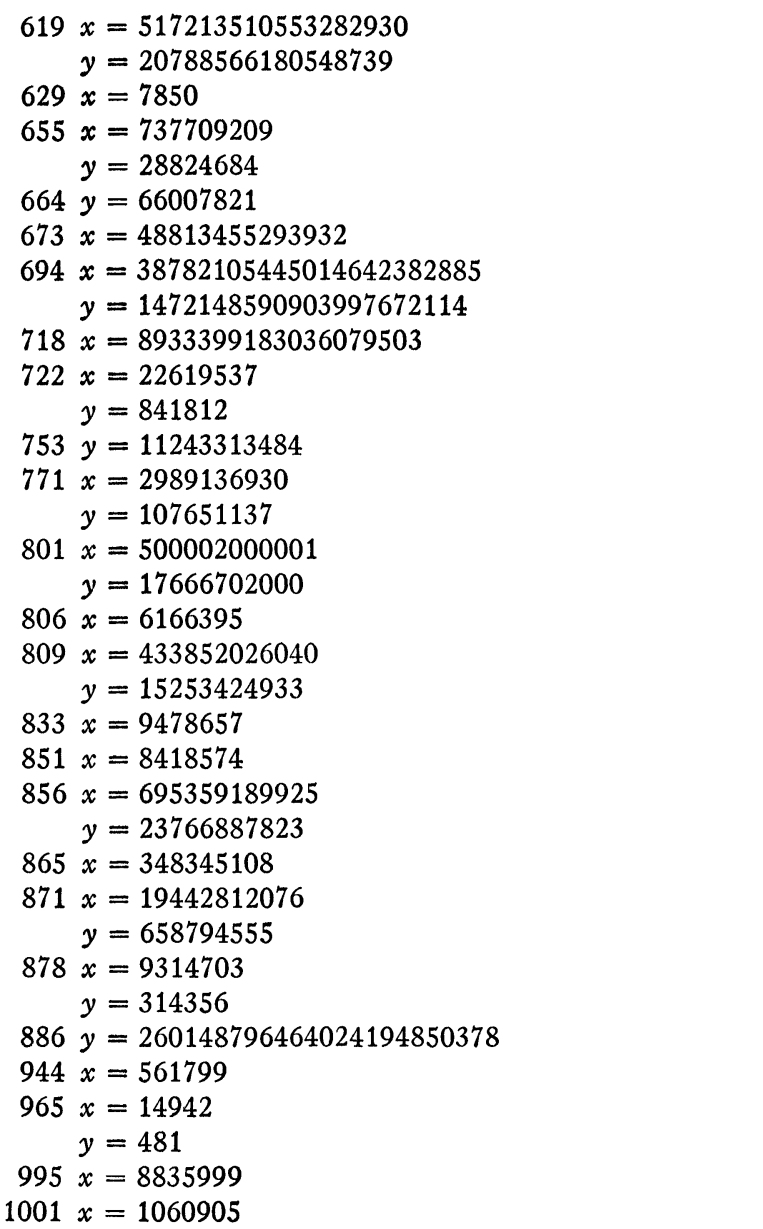

2. Degen, Canon Pellianus, Table 1.

A

$238 y=756$

$277 x=159150073798980475849$

$421 y=189073995951839020880499780706260$

$437 x=4599$

$613 y=18741545784831997880308784340$

$641 x=2609429220845977814049$

$y=103066257550962737720$ 
$A$

\section{Degen, Canon Pellianus-continued}

$653 x=10499986568677299849$

$672 x=337$

$751 x=7293318466794882424418960$

$823 x=235170474903644006168$

$919 y=147834442396536759781499589$

$945 x=275561$

$949 y=19789181711517243032971740$

$951 x=224208076$

3. Legendre, $3 \mathrm{~d}$ and 4 th editions, Table $\mathrm{x}$.

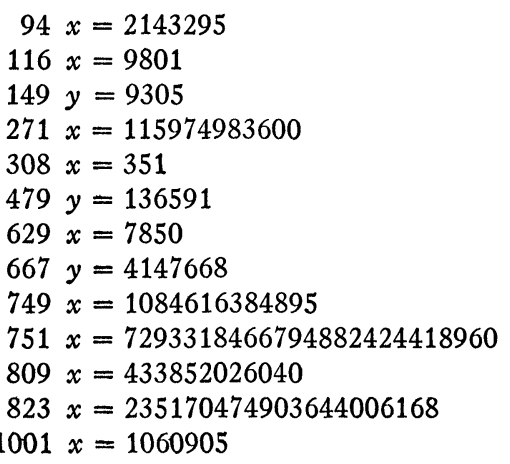

4. Bickmore, British Association Report, 1893.

$1014 y=146246$

$1366 y=61987877112128467931286485364042$

5. Whitford, The Pell Equation.

$1549 y=12223095428267474959342426833463805$ 08818076263178681966098672827963220

$1566 y=308792110$

$1615 y=81732$

$1669 y=572847173280387374124056899880229$ 34138392598249664340

The University of California 